\title{
Suitability of tunable Fabry-Perot spectrometers for condition monitoring purposes of gear oils in offshore wind turbines.
}

\author{
Benjamin R. Wiesent, Daniel D. Dorigo, Alexander W. Koch \\ Technische Universität München / Lehrstuhl für Messsystem- und Sensortechnik \\ Theresienstraße 90 / N5, 80333 München
}

\section{Abstract}

The offshoring of wind turbines is an important factor for increasing the regenerative part of future energy generation. The turbines are getting larger and are often inaccessible for preventive maintenance during long periods of the year.

Measuring the oil condition gives important information on gear health and helps to protect machinery from damage. Benefits of such measurement systems are cost savings due to an enhanced service life of the lubricant, increased lead time for maintenance and oil changes that are conducted rather by oil condition than by fixed scheduled intervals. An infrared transmission spectrum of the oil sample provides important information of its condition. Such a measurement is usually performed at a high spectral resolution of $1 \mathrm{~cm}^{-1}$ covering a broad spectral range of 4000 to $500 \mathrm{~cm}^{-1}$. Continuous online monitoring by FTIR for series-application is expensive and bulky equipment is required. Miniaturized, tunable FabryPerot spectrometers (FPS) have been demonstrated to perform well in gas analysis applications and have the potential for the construction of a cost effective infrared oil sensor.

In this paper the suitability of miniaturized, tunable FPSs for condition monitoring of wind turbines is analyzed. Two commercial FPSs LFP-3041L and LFP-3950L from InfraTec are analyzed for their applicability to this measurement problem. A third notional FP spectrometer based on a tandem setup of two tunable etalons is presented and its performance for the detection of oil aging is evaluated.

Based on a representative data set of used oils, spectral bandwidth and resolution of the spectra are limited to the properties of the FP based mini spectrometers. The original data set, taken by a FTIR with a resolution of $1 \mathrm{~cm}^{-1}$ covers the spectral range from 4000 to $500 \mathrm{~cm}^{-1}$, the second dataset covers the range from 3333 to $2439 \mathrm{~cm}^{-1}$, data set three covers the range from 2564 to $2083 \mathrm{~cm}^{-1}$ corresponding to InfraTec LFP 3041L and LFP-3950L respectively. Data set four covers the range 1818 to $909 \mathrm{~cm}^{-1}$ corresponding to the simulated Fabry-Perot Tandem Spectrometer (FPTS) approach.

Out of these downscaled spectra a partial least square regression (PLS) was done to predict important oil quality parameters like total acid number (TAN) and additive depletion. Prediction quality of these parameters is used to evaluate the usability of the respective mini spectrometer to the oil monitoring application.

\section{Introduction}

Offshore wind turbines have the potential of generating renewable energy on a large scale. Currently several offshore wind farms are under construction all over the world. The used turbines are in the $5 \mathrm{MW}$ class and beyond and often feature a large planetary gear with more than 1000 liters of lubricating oil. Modern onshore wind turbines (WTs) have an availability of about 95\% to 99\% [1]. However, there are still a number of faults that cause unscheduled downtimes. These downtimes lead to high maintenance efforts and production losses. In the German scientific measurement and evaluation program (WMEP) operation \& maintenance (O\&M) and repair reports from 1500 WTs in the period from 1989 - 2006 [2] were analyzed. According to the WMEP onshore statistics, the gearbox has the highest downtime per failure (more than six days). This downtime is expected to be even higher offshore cause of the rough environmental conditions and the limited accessibility of offshore wind farms. By monitoring important oil quality parameters wear dependent maintenance becomes possible and a longer warning time is achieved.

\subsection{IR Oil condition monitoring}

Infrared (IR) oil analysis is a de facto standard in laboratory oil analysis programs. Several publications show the successful prediction of oil quality parameters by use of high resolution and high bandwidth FTIR spectra [3-5]. A principal component analysis (PCA) followed by partial least square regression (PLS) is used to predict oil quality parameters out of their IR absorption spectra [6]. Parameters like 
oxidation, total acid number (TAN), additive depletion or water content can be analyzed. For condition monitoring purposes in wind turbines the installation of a FTIR is due to cost and space reasons not possible. In general IR based condition monitoring systems can be realized by reduction of resolution and bandwidth. Here two approaches can be differentiated. A dispersive and a non-dispersive (ND) solution.

\subsection{Non-dispersive (ND) sensor systems}

In ND sensor systems, narrow bandpass filters combined with an IR detector are used to sample distinct spectral points. In [7] a quad channel thermopile detector, equipped with four narrow bandpass filters was used in an online condition monitoring system for marine diesel engines. Agoston et. al. showed an one channel IR absorption sensor for the determination of engine oil deterioration [8]. In this approach a single element thermopile detector was used and the two narrow bandpass filters were alternately set in the light path. Kudlaty et. al. showed an IR absorption sensor employing attenuated total reflection (ATR) $[9,10]$. Here a single element pyroelectric detector and a rotating chopper disc with integrated narrow bandpass filters were used.

ND sensor systems are of low-cost, robust against vibration, and perform well in some special purpose applications.

\subsection{Dispersive sensor systems}

A more general approach for oil condition monitoring is the use of a dispersive element like e.g. a grating or a linear variable filter (LVF) [11]. Compared to FTIR, resolution and bandwidth of dispersive oil condition monitoring sensors is limited due to cost and size. The pyroelectric detectors with integrated tunable Fabry-Perot filter from InfraTec represent a promising class of miniaturized IR spectrometers. The two available detectors have a tunable spectral range of $1.3 \mu \mathrm{m}$ at a resolution $\Delta \lambda$ of about $100 \mathrm{~nm}$. The main advantage of dispersive sensor systems is the capability of analyzing chemical substances characterized by adjacent or overlapping absorption bands [12].

For the application as oil monitoring system dispersive IR oil sensors can be adapted to the respective type of oil just by adapting the calibration without the need of changing the hardware.

\section{Materials and methods}

\subsection{Fabry-Perot spectrometers}

A Fabry-Perot interferometer is the core of this type of IR miniature spectrometers and consists of two highly reflective coplanar mirrors. Both reflectors are separated by distance $d$ and the gap between, the cavity is filled with a material of refractive index $n$. Varying the separation distance or the refractive index of the cavity results in a tuning of the spectrometer. The transmission of a Fabry-Perot configuration is described by the Airy function [12-14].

$$
\mathrm{A}(\mathrm{d}, \lambda, \mathrm{n}, \theta)=\frac{\mathrm{T}}{1+\left(\frac{2 \mathrm{~F}_{\mathrm{R}}}{\pi}\right)^{2} \sin ^{2}\left(\frac{2 \pi \mathrm{nd} \cos \theta}{\lambda}\right)}
$$

The transmission of the Airy function depends on four variables: $d$ the separation distance, $\mathrm{n}$ the refractive index of the cavity, $\lambda$ the wavelength, and $\theta$ the angle of incidence. $F_{R}$ is the single-pass reflectivity finesse and $T$ the single-pass transmission.

$$
\begin{gathered}
\mathrm{F}_{\mathrm{R}}=\frac{\pi \mathrm{R}}{\sqrt{(1-\mathrm{R})}} \\
\mathrm{T}=\left(\frac{1-\mathrm{A}}{1-\mathrm{R}}\right)^{2}
\end{gathered}
$$

$R$ denotes the reflectivity of the mirrors and $A$ the absorption. The reflectors of InfraTec's Fabry-Perot filters are made of dielectric multi layer stacks (Bragg reflectors) [15].

The period of the Airy function is termed free spectral range (FSR). The full-width at half-maximum (FWHM) describes the bandwidth of the interference peak where half of the power is attenuated.

The key parameters for both analyzed tunable FPSs $[16,17]$ are summarized in Tab. 1. 
Tab. 1: Specifications of tunable Fabry-Perot filters LFP 3041L - 337 and LFP 3950L - 337 from InfraTec

\begin{tabular}{lll}
\hline & LFP 3041L & LFP 3950L \\
\hline Tuning range $(\boldsymbol{\mu m})$ & $3.0-4.1$ & $3.9-4.8$ \\
Tuning range $\left(\mathbf{c m}^{-1}\right)$ & $3333-2439$ & $2564-2083$ \\
FWHM $(\mathbf{n m})$ & $60-100$ & $80-120$ \\
FWHM $\left(\mathbf{c m}^{-1}\right)$ & & \\
Cavity tuning range $(\mu \mathrm{m})$ & $1.5-2.5$ & $1.5-2.5$ \\
Order number & 1 & 1 \\
Reflectivity R & $\geq 0.9$ & $\geq 0.9$ \\
\hline
\end{tabular}

\subsection{Multivariate data analysis}

To evaluate the usability of the tunable Fabry-Perot devices two common wind turbine gear oils, a mineral and a synthetic oil were used. The MID-IR spectra of 200 samples of used oil of both types were measured by a FTIR using a resolution of $1 \mathrm{~cm}^{-1}$ at the range 4000 to $500 \mathrm{~cm}^{-1}$. These spectra were analyzed by a statistical factor analysis using The Unscrambler ${ }^{\circledR}$ from Camosoft. 100 spectra were chosen to provide the calibration set. 50 of the remaining 100 samples formed the validation set.

For the mineral oil the total acid number (TAN) was used as dependent variable. During the service life of the lubricant acidic products are formed [18]. Thus the alkaline reserve or acid content of a degraded lubricant is indicated in the TAN. Especially for mineral oils TAN is an important parameter indicating the lubricant condition. For the synthetic oil the level of molybdenum was used as dependent variable. The level of molybdenum indicates deterioration of the additive pack. Lubricants are formulated from a base oil mixture and an additive pack [18]. Additives help to protect e.g. from oxidation, wear or corrosion.

Primary both dependent variables, TAN and molybdenum are predicted using high quality FTIR spectra of both oils. Next all spectra are reduced to the specific resolution and range of InfraTec tunable Fabry-Perot detectors LFP 3041L - 337 and LFP 3950L - 337 respectively. Subsequently a PLS is performed and prediction quality is compared to that using FTIR spectra.

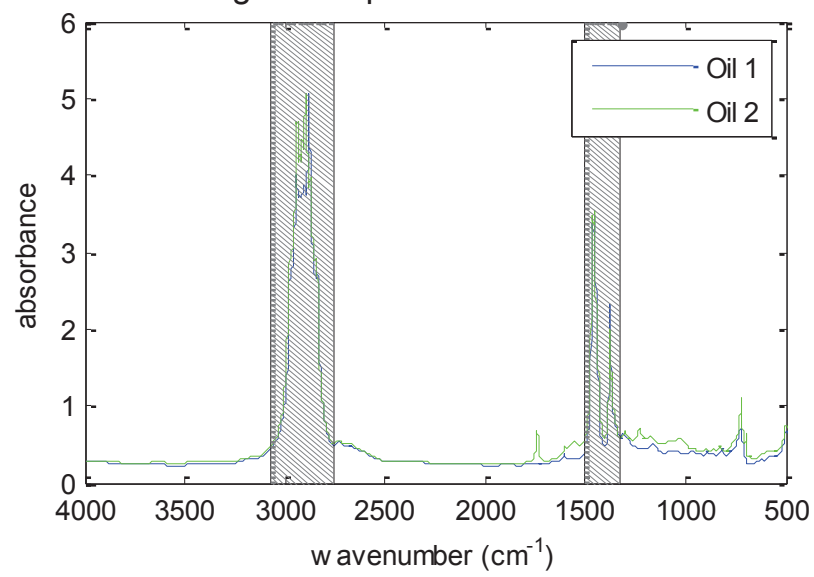

Fig. 1: IR absorption spectra of two popular wind turbine gear oils. The fresh oil spectra were measured at a resolution of $1 \mathrm{~cm}-1$. The hatched areas mark unused spectral ranges due to non-linearity of the Lambert-Beer law.

Fig. 1 shows the fresh oil spectra of two popular wind turbine gear oils. Oil 1 is a mineral lubricating oil and Oil 2 is a synthetic lubricant. The hatched areas mark unused spectral ranges from 3067 to $2758 \mathrm{~cm}^{-1}$ and 1500 to $1330 \mathrm{~cm}^{-1}$. These areas have to be blanked out for further factor analysis because of the non-linearity of the Lambert-Beer law at absorbance exceeding a value of 2.5.

A Prediction model, generated by multivariate data analysis, looks for changes within the sample set of modeling spectra and relates these changes to the dependent parameter being predicted. This prediction model contains the variations within the spectra that relates to the modeled parameter. The regression coefficient contains both positive and negative spectral changes that relate to changes in the dependent variable. 

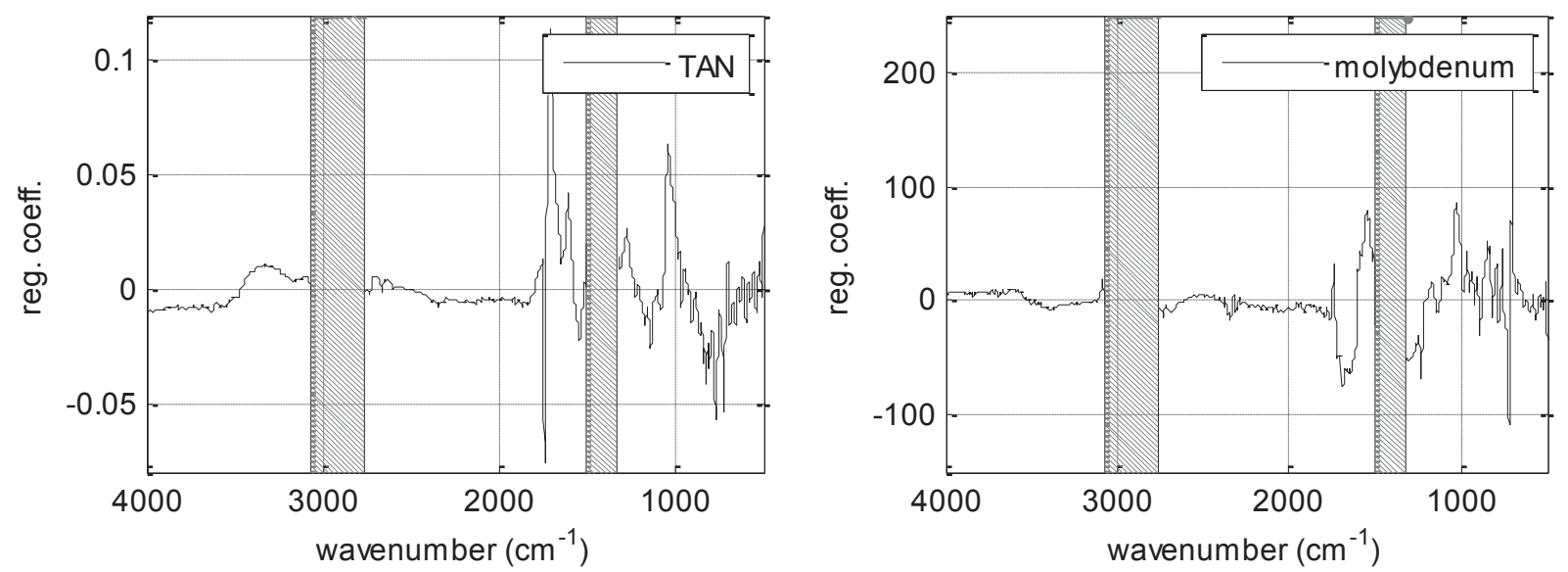

Fig. 2: Regression coefficient of TAN for Oil 1and molybdenum levels for Oil 2

Tab. 2 shows the regression coefficient of TAN for Oil 1 and molybdenum for Oil 2. Both plots indicate the contribution of spectral changes to the prediction. It can be seen that for both dependent variables information carrying structures start at a wavenumber of about $1800 \mathrm{~cm}^{-1}$. Both tunable Fabry-Perot spectrometers operate in spectral regions with a minor or almost no contribution to prediction.

\subsection{Extension of the spectral range}

To obtain a wider spectral range an arrangement of two Fabry-Perot resonators, aligned in series are a possible solution. With a spectral range starting at 1800 up to $900 \mathrm{~cm}^{-1}$ a large portion of the important part of the regression coefficient is covered. There are two configurations to arrange two Fabry-Perot resonators in series. One is called the multiple integer tandem arrangement. Here the spacing of the first Fabry-Perot is set as an exact multiple of the other [19]. For example the Fabry-Perot resonator one is operated at an order of one and the second at an order of two. The second possible arrangement is called vernier tandem. Here the two cavity length are made slightly different with respect to each other $[14,19,20]$.

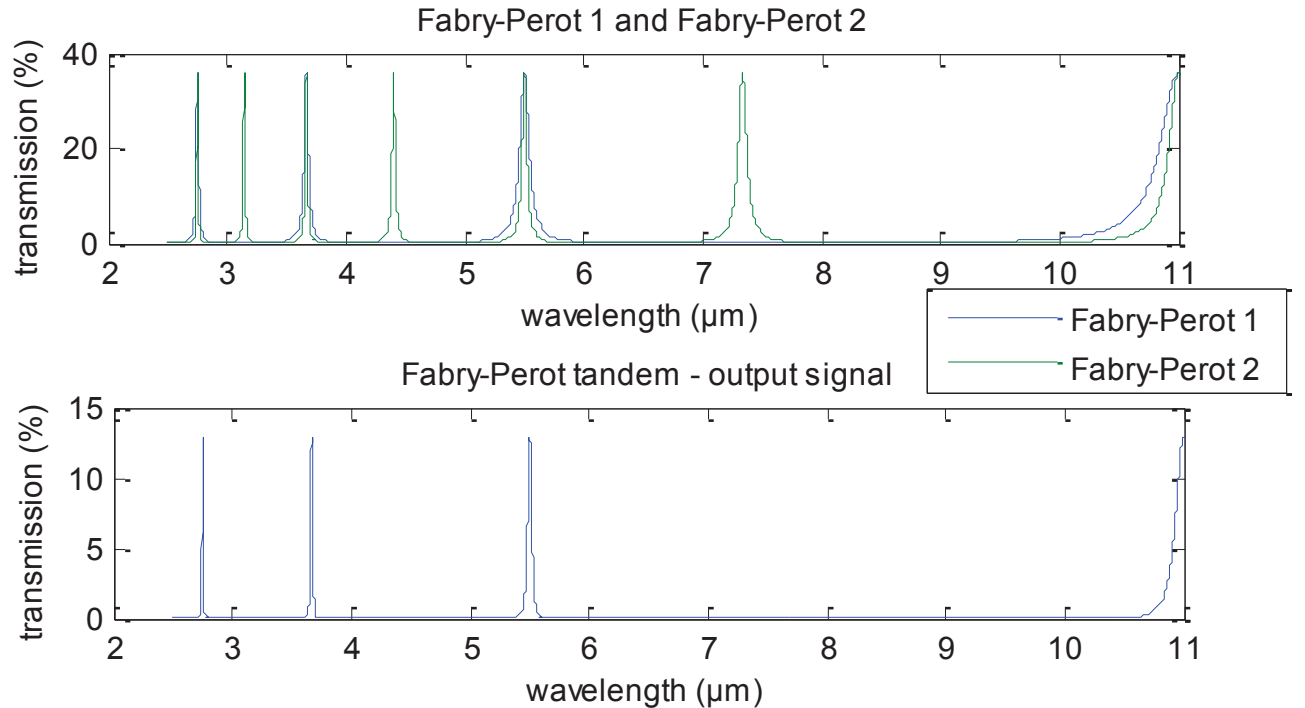

Fig. 3: Fabry-Perot 1 is operated in order 1 and Fabry-Perot 2 is operated in order 2. Fabry-Perot tandem shows the output signal of both Fabry-Perots aligned in series.

According to Fig. 3 the tandem arrangement meets the analyzed requirements. The FSR is extended to $5,5 \mu \mathrm{m}$. By replacing the single channel detector with a multichannel one, covered by thin film band-pass filters the spectral range of such a tandem Fabry-Perot could be enlarged even more.

For the simulation a reflectivity $R$ of 0.9 and an absorption $A$ of 0.04 [19] was used. Thus a system transmissivity of $12 \%$ was obtained. Further the phase shift in reflections at the etalon surface was neglected, the refractive index $\mathrm{n}$ was set to 1 assuming air as fill gas and the angle of incidence $\theta$ was set to 0 . For subsequent analysis a single detector tandem Fabry-Perot with a scanning range of 5.5 to 11 $\mu \mathrm{m}$ was used. The tuning range of the two cavities were 2.75 to $5.5 \mu \mathrm{m}$ for FP1 and 5.5 to $11 \mu \mathrm{m}$ for FP2 
respectively. The resolution of this TFPS was calculated to be $24 \mathrm{~cm}^{-1}$ at $1818 \mathrm{~cm}^{-1}$ and $13 \mathrm{~cm}^{-1}$ at $909 \mathrm{~cm}^{-1}$.

\section{Results and discussion}

To evaluate the suitability of the three FPSs prediction qualities of both important oil quality parameters, TAN and molybdenum level are compared to the FTIR model. Tab. 2 gives an overview of the PLS prediction statistics.

Tab. 2: Statistics for factor analysis of Oil 1 (TAN) and Oil 2(molybdenum) using spectra of FTIR, InfraTec LFP3041L-337, LFP-3950L-337 and a TFPS

\begin{tabular}{|c|c|c|c|c|}
\hline & & 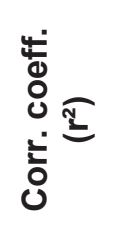 & 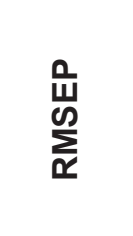 & 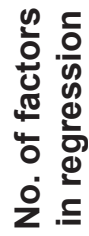 \\
\hline \multirow[t]{2}{*}{ FTIR } & $\begin{array}{c}\text { TAN } \\
\text { (mgKOH/g) }\end{array}$ & 0.899 & 0.020 & 6 \\
\hline & $\begin{array}{l}\text { Molybdenum } \\
\text { (mg/kg) }\end{array}$ & 0.875 & 92.62 & 5 \\
\hline \multirow[t]{2}{*}{ LFP-3041L-337 } & $\begin{array}{c}\text { TAN } \\
\text { (mgKOH/g) }\end{array}$ & 0.256 & 0.063 & 6 \\
\hline & $\begin{array}{l}\text { Molybdenum } \\
\text { (mg/kg) }\end{array}$ & 0.647 & 185.55 & 5 \\
\hline \multirow[t]{2}{*}{ LFP-3950L-337 } & $\begin{array}{c}\text { TAN } \\
\text { (mgKOH/g) }\end{array}$ & 0.214 & 0.065 & 6 \\
\hline & $\begin{array}{l}\text { Molybdenum } \\
\text { (mg/kg) }\end{array}$ & 0.350 & 251.02 & 5 \\
\hline \multirow[t]{2}{*}{ TFPS } & $\begin{array}{c}\text { TAN } \\
\text { (mgKOH/g) }\end{array}$ & 0.838 & 0.023 & 6 \\
\hline & $\begin{array}{l}\text { Molybdenum } \\
\text { (mg/kg) }\end{array}$ & 0.854 & 154.15 & 5 \\
\hline
\end{tabular}

Tab. 2 lists the results of PLS regression of Oil 1 (TAN) and Oil 2 (molybdenum) using FTIR and FPS spectra. Statistics of FTIR spectra for prediction of TAN and molybdenum levels show high quality with a regression coefficient $r^{2}$ of 0.899 and 0.875 respectively. The prediction quality of both FPSs is rather poor, with $r^{2}$ of 0.256 and 0.214 respectively for TAN as dependent variable. For the prediction of molybdenum levels the LFP3041-337L shows a medium quality $\mathrm{r}^{2}$ of 0.647 . However, the LFP-3950-337L gives a rather poor correlation of 0.350 . The FPTS shows good prediction quality for both dependent variables with $r^{2}$ of 0.838 for TAN and 0.854 for molybdenum levels. Both correlation coefficients are a bit worse than the FTIR results but are still usable for oil condition monitoring purposes. For this application the covered spectral range is more important than the spectral resolution.

\section{Conclusion}

In this study the suitability of band and resolution limited IR spectra of InfraTec tunable Fabry-Perot spectrometers LFP-3041L-337 and LFP-3950L-337 with application to oil condition monitoring was analyzed. Two important oil quality parameters, TAN and additive depletion were predicted using multivariate statistics and IR spectra. Two popular wind turbine gear oils, a mineral and a synthetic one were used to validate the practicability of both available FPS. A third notional FPTS was adjusted to fit the measurement problem. TFPs mini spectrometers in combination with sophisticated multivariate data processing are a possibility for an in-situ oil condition monitoring sensor.

However both available FPSs operate in a wavelength range that is not appropriate. For the prediction of molybdenum levels in synthetic oils, LFP-3041L-337 shows a moderate prediction quality. The FPTS approach, adjusted to the wavelength range 1818 to $909 \mathrm{~cm}^{-1}$ showed good prediction performance. The spectral resolution of first order FPSs was sufficient for the prediction of TAN levels in mineral based oils and additive depletion in synthetic gear oils. 


\section{List of References}

[1] S. Faulstich, B. Hahn, H. Jung, and K. Rafik, "Suitable failure statistics as a key for improving availability," Proceedings of the European Wind Energy Conference, EWEC, 2009.

[2] S. Faulstich, M. Durstewitz, B. Hahn, K. Knorr, and K. Rohrig, "Windenergie Report Deutschland 2008," Institut für solare Energieversorgungstechnik (Hrsg.), Kassel, 2008.

[3] M. Zakharich, I. Zaitsev, V. Komar, F. Nikonovich, M. Ryzhkov, and I. Skornyakov, "Analysis of Transformer Oil Using IR Analyzers," Journal of Applied Spectroscopy, vol. 68, 2001, p. 61-65.

[4] F. Van De Voort, J. Sedman, R. Cocciardi, and D. Pinchuk, "FTIR Condition Monitoring of InService Lubricants: Ongoing Developments and Future Perspectives," Tribology Transactions, vol. 49, Sep. 2006, pp. 410-418.

[5] A.M. Toms, "FOURIER-TRANSFORM INFRARED (FTIR) SPECTROSCOPY-APPLYING THE CORRECT METHOD FOR YOUR APPLICATION," gastopsusa.com, 2007, pp. 10-12.

[6] A.D. Stuart, S.M. Trotman, K.J. Doolan, and P.M. Fredericks, "Spectroscopic Measurement of Used Lubricating Oil Quality," Applied Spectroscopy, vol. 43, Jan. 1989, pp. 55-60.

[7] L. Ben Mohammadi, F. Kullmann, M. Holzki, S. Sigloch, T. Klotzbuecher, J. Spiesen, T. Tommingas, P. Weismann, and G. Kimber, "A low cost mid-infrared sensor for on line contamination monitoring of lubricating oils in marine engines," Measurement, 2010, p. 77260M$77260 \mathrm{M}-12$.

[8] A. Agoston, C. Oetsch, J. Zhuravleva, and B. Jakoby, "An IR-absorption sensor system for the determination of engine oil deterioration," Proceedings of IEEE Sensors, 2004., 2004, pp. 463466.

[9] K. Kudlaty, A. Purde, and A. Koch, "Development of an Infrared Sensor for On-line Analysis of Lubricant Deterioration," 2nd IEEE International Conference on Sensors, New York, 2003, p. 903908.

[10] K. Kudlaty and A.W. Koch, "In-situ-Analyse von Schmierölalterung mittels ATR-IR- Sensorik," VDI Tagung "Sensoren und Messsysteme 2004", 2004.

[11] B.R. Wiesent, D.G. Dorigo, and A.W. Koch, "Limits of IR-spectrometers based on linear variable filters and detector arrays," Proceedings of SPIE, 2010, p. 77670L.

[12] N. Neumann, "Tunable infrared detector with integrated micromachined Fabry-Perot filter," Journal of Micro/Nanolithography, MEMS and MOEMS, vol. 7, 2008, p. 021004.

[13] F.L. Pedrotti, L. Pedrotti, W. Bausch, and H. Schmidt, Optik für Ingenieure, Springer, 2005.

[14] A. Yoshihara, "Construction of a Vernier Tandem (3+3)-Pass Brillouin Spectrometer and its Application to Surface Acoustic Wave Study in Metallic Multilayer Films," Jpn. J. Appi. Phys. Vol, vol. 33, 1994, p. 3100-3109.

[15] InfraTec, "Advanced Features of InfraTec Pyroelectric Detectors," In Practice, vol. 1, 2010, pp. 110.

[16] InfraTec, "Datasheet LFP-3041L-337- \# pyroelectric detector with tunable FPF," 2010, p. 2.

[17] InfraTec, "Datasheet LFP-3950L-337- \# pyroelectric detector with tunable FPF," 2010, p. 2.

[18] R.M. Cresham and G.E. Totten, Lubrication \& Maintenance of Industrial Machinery, CRC Press Taylor \& Francis Group, 2009.

[19] J.M. Chalmers and P.R. Griffiths, "Handbook of vibrational spectroscopy," Instrumentation, 2002.

[20] S. Lindsay, M. Anderson, and J. Sandercock, "Construction and alignment of a high performance multipass vernier tandem Fabry-Perot interferometer," Review of Scientific Instruments, vol. 52, 2009, p. 1478-1486. 